\title{
Intrinsic spectra and energetics of BeppoSAX Gamma-Ray Bursts with known redshifts
}

\author{
L. Amati ${ }^{1}$, F. Frontera ${ }^{1,2}$, M. Tavani ${ }^{3}$, J. J. M. in 't Zand ${ }^{4}$, A. Antonelli ${ }^{5}$, E. Costa ${ }^{6}$, M. Feroci ${ }^{6}$, C. Guidorzi ${ }^{2}$,

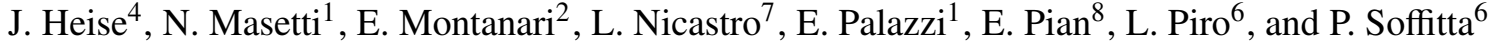 \\ ${ }^{1}$ Istituto di Astrofisica Spaziale e Fisica cosmica - Sezione di Bologna, CNR, Via Gobetti 101, 40129 Bologna, Italy \\ 2 Dipartimento di Fisica, Università di Ferrara, Via Paradiso 12, 44100 Ferrara, Italy \\ 3 Istituto di Astrofisica Spaziale e Fisica cosmica - Sezione di Milano, CNR, Via Bassini 15, 20133 Milano, Italy \\ 4 Space Research Organization Netherlands, Sorbonnelaan 2, 3584 CA Utrecht, The Netherlands \\ 5 Osservatorio Astronomico di Roma, Via Frascati 33, 00040 Monteporzio Catone (RM), Italy \\ ${ }^{6}$ Istituto di Astrofisica Spaziale e Fisica cosmica, CNR, Via Fosso del Cavaliere, 00133 Roma, Italy \\ 7 Istituto di Astrofisica Spaziale e Fisica cosmica - Sezione di Palermo, CNR, Via La Malfa 153, 90146 Palermo, Italy \\ 8 Osservatorio Astronomico di Trieste, Via G.B. Tiepolo 11, 34131, Trieste, Italy
}

Received 6 February 2001 / Accepted 15 May 2002

\begin{abstract}
We present the main results of a study of spectral and energetics properties of twelve gamma-ray bursts (GRBs) with redshift estimates. All GRBs in our sample were detected by BeppoSAX in a broad energy range (2-700 keV). From the redshift estimates and the good-quality BeppoSAX time-integrated spectra we deduce the main properties of GRBs in their cosmological rest frames. All spectra in our sample are satisfactorily represented by the Band model, with no significant soft $\mathrm{X}$-ray excesses or spectral absorptions. We find a positive correlation between the estimated total (isotropic) energies in the 1-10000 keV energy range $\left(E_{\mathrm{rad}}\right)$ and redshifts $z$. Interestingly, more luminous GRBs are characterized also by larger peak energies $E_{\mathrm{p}} \mathrm{s}$ of their $E F(E)$ spectra. Furthermore, more distant GRBs appear to be systematically harder in the X-ray band compared to GRBs with lower redshifts. We discuss how selection and data truncation effects could bias our results and give possible explanations for the correlations that we found.
\end{abstract}

Key words. gamma-rays: bursts - gamma rays: observations - X-rays: general

\section{Introduction}

In this paper we report and discuss the main spectral properties of the X- gamma-ray emission from GammaRay Bursts (GRBs) with known redshift detected by the BeppoSAX satellite (Boella et al. 1997). We base our work on data obtained from the co-aligned wide field detectors on board BeppoSAX: the Gamma Ray Burst Monitor (GRBM, 40-700 keV, Frontera et al. 1997) and the two Wide Field Cameras (WFC, 2-28 keV, Jager et al. 1997). The combination of these detectors makes possible not only localizations for GRBs occurring in the WFC $20^{\circ} \times 20^{\circ}(F W H M)$ field of view, but also reliable estimates of their spectra from 2 to $\sim 700 \mathrm{keV}$ (e.g. Frontera et al. 2000).

Until now, 42 GRBs have been simultaneously detected by the BeppoSAX WFC and GRBM. For twelve of these events, reliable redshift estimates are available (see Table 1 and references therein), allowing the investigation of systematic trends between the GRBs spectral parameters and either GRB redshift or total radiated energy. The extension of the spectral

Send offprint requests to: L. Amati, e-mail: amati@tesre.bo.cnr.it analysis to the $\mathrm{X}$-ray energy band allows a better determination of the continuum GRB spectrum, reducing the bias in the measurement of the spectral slope below the peak energy $E_{\mathrm{p}}$ of the $E F(E)$ spectrum. Our analysis is based on GRB time-integrated spectra. Compared to the time-resolved spectra available for several of our GRBs, the spectra considered in this paper have a good statistical quality and are less affected by possible "absorption effects" expected and observed at the very early times (Frontera et al. 2000; Amati et al. 2000).

\section{The GRB sample and redshift estimates}

Table 1 summarizes the basic $\mathrm{X}$ - and gamma-ray observed properties of the GRBs included in our sample. For six events (GRB 970228, GRB 970508, GRB 971214, GRB 980613, GRB 990705 and GRB 990712) redshifts are available from optical emission lines in the spectrum of their host galaxies. For GRB 990123, GRB 990510, and GRB 010222, lower limits are available from the detection of absorption lines in optical transient (OT) spectra. These lower limits are reliable distance indicators: when redshift measurements from both absorption and emission lines are available, it is found that they are 
Table 1. Observed physical parameters of GRBs included in our sample.

\begin{tabular}{llllllllll}
\hline \hline GRB & $\begin{array}{l}P_{\mathrm{X}}{ }^{a} \\
(2-28 \mathrm{keV})\end{array}$ & $\begin{array}{l}S_{\mathrm{X}}{ }^{b} \\
(2-28 \mathrm{keV})\end{array}$ & $\begin{array}{l}\Delta T_{\mathrm{X}}{ }^{c} \\
(\mathrm{~s})\end{array}$ & $\begin{array}{l}P_{\gamma}{ }^{a} \\
(40-700 \mathrm{keV})\end{array}$ & $\begin{array}{l}S_{\gamma}{ }^{b} \\
(40-700 \mathrm{keV})\end{array}$ & $\begin{array}{l}\Delta T_{\gamma}{ }^{c} \\
(\mathrm{~s})\end{array}$ & $\begin{array}{l}S_{\gamma, \text { min }}{ } \\
\text { Redshift } z \\
e\end{array}$ & $\begin{array}{l}\text { Ref. } \\
f\end{array}$ \\
\hline 970228 & $0.25 \pm 0.03$ & $3.2 \pm 0.2$ & 75 & $3.7 \pm 0.1$ & $11 \pm 1$ & 80 & 0.20 & $0.695(\mathrm{HG})$ & $(1)$ \\
970508 & $0.050 \pm 0.011$ & $0.83 \pm 0.04$ & 25 & $0.34 \pm 0.01$ & $1.8 \pm 0.3$ & 20 & 0.29 & $0.835(\mathrm{OT}+\mathrm{HG})$ & $(2)$ \\
971214 & $0.060 \pm 0.009$ & $0.32 \pm 0.05$ & 40 & $0.68 \pm 0.07$ & $8.8 \pm 0.9$ & 35 & 0.70 & $3.42(\mathrm{HG})$ & $(3)$ \\
980326 & $0.15 \pm 0.03$ & $0.55 \pm 0.08$ & 9 & $0.245 \pm 0.015$ & $0.75 \pm 0.15$ & 9 & 0.09 & $0.9-1.1(\mathrm{PH})$ & $(4)$ \\
980329 & $0.21 \pm 0.07$ & $4.3 \pm 0.3$ & 40 & $3.1 \pm 0.1$ & $65 \pm 5$ & 25 & 0.75 & $2.0-3.9(\mathrm{PH})$ & $(5)$ \\
980613 & $0.019 \pm 0.006$ & $0.27 \pm 0.05$ & 40 & $0.16 \pm 0.04$ & $1.0 \pm 0.2$ & 20 & 0.14 & $1.096(\mathrm{HG})$ & $(6)$ \\
990123 & $0.21 \pm 0.02$ & $9.0 \pm 0.1$ & 100 & $17.0 \pm 5.0$ & $300 \pm 40$ & 100 & 1.18 & $1.6(\mathrm{OT})$ & $(7)$ \\
990510 & $0.17 \pm 0.03$ & $5.5 \pm 0.2$ & 120 & $2.47 \pm 0.21$ & $19 \pm 2$ & 75 & 0.39 & $1.619(\mathrm{OT})$ & $(8)$ \\
990705 & $0.18 \pm 0.03$ & $5.4 \pm 0.2$ & 60 & $3.7 \pm 0.1$ & $75 \pm 8$ & 42 & 1.05 & $0.843(\mathrm{XP}+\mathrm{HG})$ & $(9)$ \\
990712 & $0.66 \pm 0.04$ & $5.2 \pm 0.1$ & 45 & $1.3 \pm 0.1$ & $6.5 \pm 0.3$ & 20 & 0.50 & $0.43(\mathrm{OT}+\mathrm{HG})$ & $(8)$ \\
000214 & $0.075 \pm 0.010$ & $1.7 \pm 0.1$ & 80 & $4.0 \pm 0.2$ & $14.2 \pm 0.4$ & 10 & 0.21 & $0.37-0.47(\mathrm{XA})$ & $(10)$ \\
010222 & $0.47 \pm 0.04$ & $21.0 \pm 0.3$ & 280 & $8.6 \pm 0.2$ & $92.5 \pm 2.8$ & 130 & 0.55 & $1.473(\mathrm{OT})$ & $(11)$ \\
\hline
\end{tabular}

a Detected $1 \mathrm{~s}$ peak fluxes in units of $10^{-6} \mathrm{erg} \mathrm{cm}^{-2} \mathrm{~s}^{-1}$.

$b$ Detected fluences in units of $10^{-6} \mathrm{erg} \mathrm{cm}^{-2}$.

${ }^{c}$ Evaluated from the background subtracted $1 \mathrm{~s}$ GRB light curve as the difference between the times of the last and first bins with a count $\approx 3 \sigma$.

${ }^{d}$ Minimum detectable fluences (see text) in units of $10^{-6} \mathrm{erg} \mathrm{cm}^{-2}$.

$e$ OT $=$ redshift determined from absorption lines in the Optical Transient spectrum; HG $=$ redshift based on optical emission lines in the Host Galaxy spectrum; $\mathrm{PH}=$ redshift estimated from photometric data; $\mathrm{XP}=$ redshift inferred from an absorption feature in the X-ray spectrum of the prompt emission. XA = redshift inferred from an emission line in the spectrum of the X-ray afterglow.

$f$ References for the redshift measurements: (1) Bloom et al. (2000); (2) Bloom et al. (1998); (3) Kulkarni et al. (1998); (4) Bloom et al. (1999); (5) Lamb et al. (1999) and references therein; (6) Djorgovski et al. (2000); (7) Kulkarni et al. (1999); (8) Vreeswijk et al. (2000); (9) Amati et al. (2000), Andersen et al. (2002); (10) Antonelli et al. (2000); (11) e.g. Stanek et al. (2001).

coincident. For three more bursts (GRB 980326, GRB 980329 and GRB 000214) we adopt $z$ values based, respectively, on the time behavior of the optical afterglow, on optical/infrared photometric estimates and on the detection of an emission line in the X-ray afterglow spectrum (see references in Table 1). In Col. 8 of Table 1 we report for each GRB the $40-700 \mathrm{keV}$ fluence $S_{\gamma \text {,min }}$ below which the event would not have been triggered and thus detected, assuming the same duration, light curve shape and spectrum of the detected GRB and as background level that measured during the GRB observation.

As can be seen from Table 1, the GRB emission properties reported (X-gamma-ray fluence, $1 \mathrm{~s}$ peak flux and duration) vary over a broad range. The dynamic range of the peak flux and fluence is two orders of magnitude in $\gamma$-rays and one order of magnitude in X-rays. No significant correlation is found between redshift and GRBs peak flux, fluence or duration. The time profiles of GRBs in our sample are either simple or complex and the range of GRB duration is wide, from 9 to 122 seconds. All the events belong to the "long GRB class", with $T_{90}$ durations larger than $2 \mathrm{~s}$, according to the standard GRB classification. The long duration of the GRBs localized by BeppoSAX may be due to the settings of the GRBM onboard trigger logic, which favors the detection of events with $\Delta T>$ few seconds, as reported by Guidorzi (2002). Possible selection effects which could affect our sample are discussed in more detail in Sect. 5 .

\section{Data analysis method}

The spectral analysis was performed by adopting standard GRBM and WFC data reduction techniques (see Amati et al. 1999 for the GRBM and Jager et al. 1997 for the WFC). The cross-calibration of the two instruments was performed using simultaneous observations of the Crab source (Amati 1999). Given that the GRBM spectra have a time resolution of $128 \mathrm{~s}$, the effective exposure time to each event is estimated from the $1 \mathrm{~s}$ resolution light curves. The spectra were fit assuming as input model the smoothly broken power-law proposed by Band et al. (1993), whose parameters are the lowenergy index $\alpha$, the break energy $E_{0}$ (in $\mathrm{keV}$ ), the high energy index $\beta$ and the normalization parameter (at $100 \mathrm{keV}$ ) $A$, and takes the form:

$$
\begin{aligned}
& N(E)=A \cdot\left(\frac{E}{100 \mathrm{keV}}\right)^{\alpha} \cdot \exp \left(-E / E_{0}\right), \\
& N(E)=A \cdot\left[\frac{(\alpha-\beta) \cdot E_{0}}{100 \mathrm{keV}}\right]^{\alpha-\beta} \cdot \exp (\beta-\alpha) \cdot\left(\frac{E}{100 \mathrm{keV}}\right)^{\beta}
\end{aligned}
$$

for $E \leq(\alpha-\beta) \cdot E_{0}$ and for $E \geq(\alpha-\beta) \cdot E_{0}$ respectively.

In carrying out the spectral fitting we took into account the Galactic absorption along the burst directions using the photoelectric cross-sections by Morrison and Mc Cammon (1983) and the column density maps by Dickey \& Lockman (1990).

With respect to previous works (e.g. Jimenez et al. 2001; Bloom et al. 2001) we adopted a different technique for the 
GRB 970228

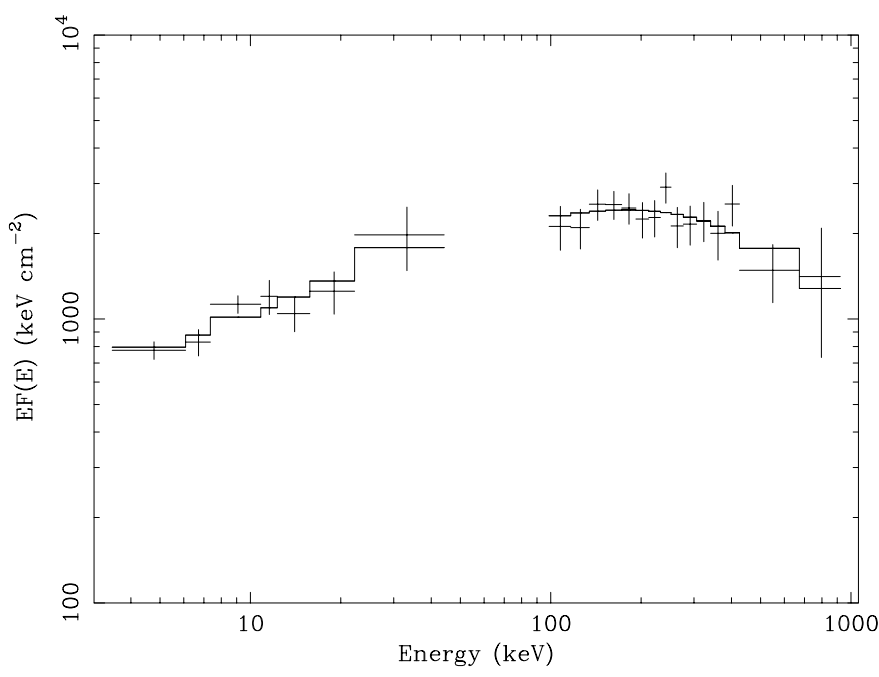

GRB 971214

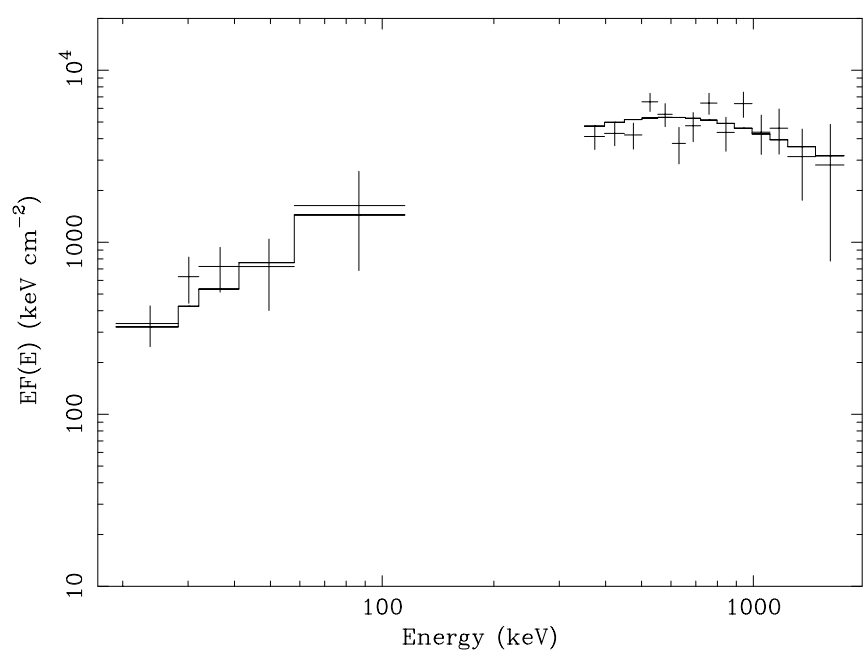

GRB 980329

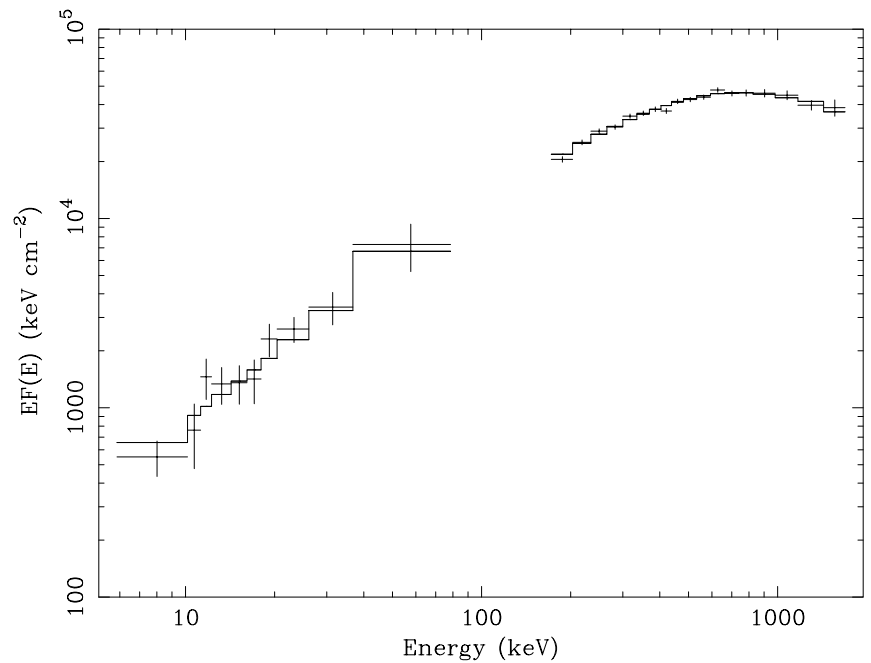

GRB 970508

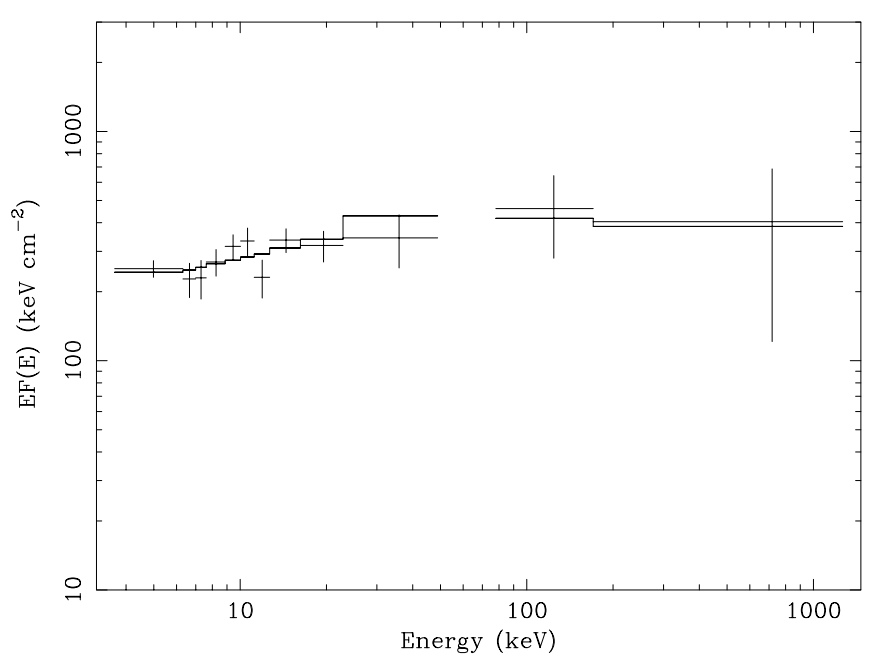

GRB 980326

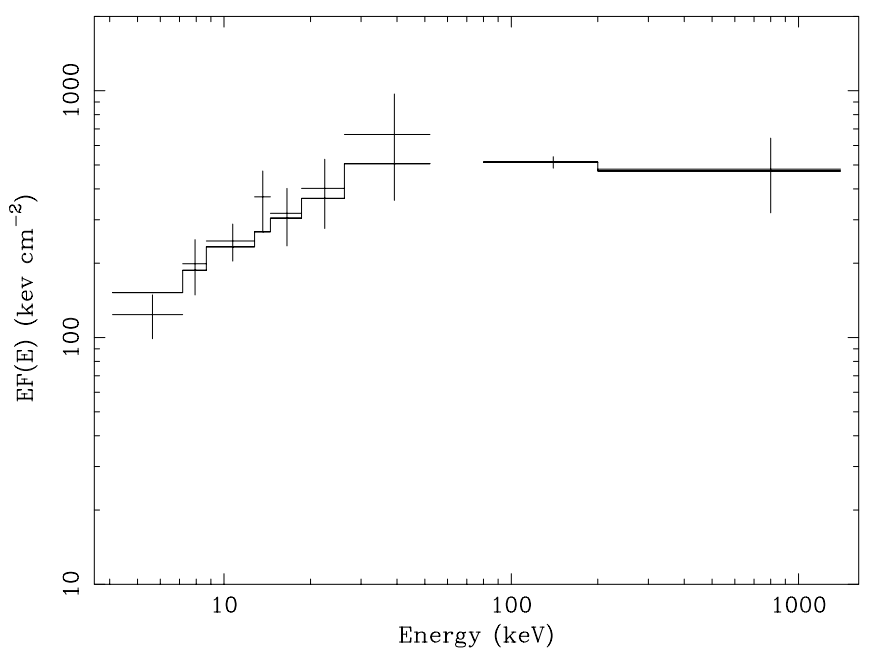

GRB 980613

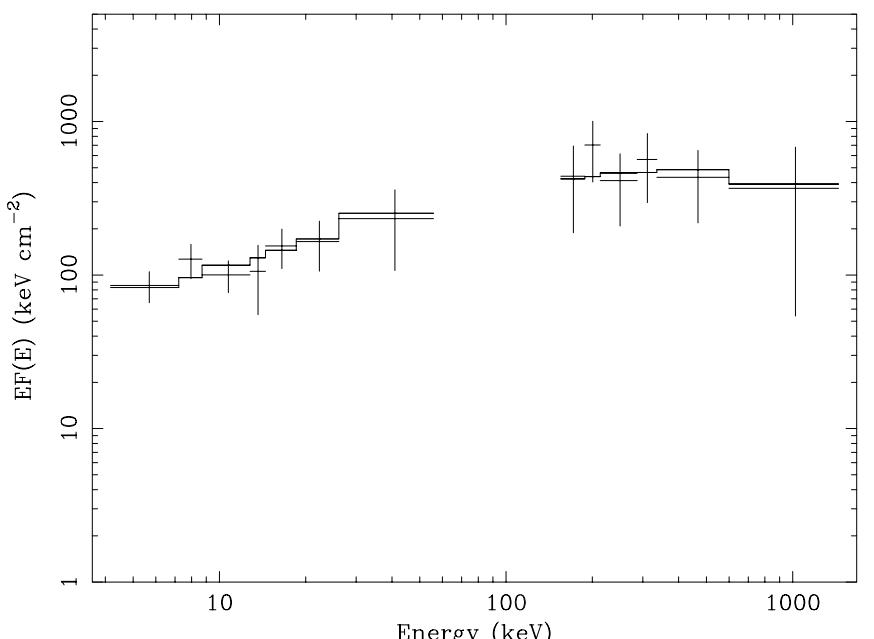

Fig. 1. Intrinsic time-integrated $E F(E)$ spectra of the GRBs included in our sample. WFC data: low-energy data-set; GRBM data: at higher energies. Continuous line: best fit curve with the Band law (see Table 2). 
GRB 990123

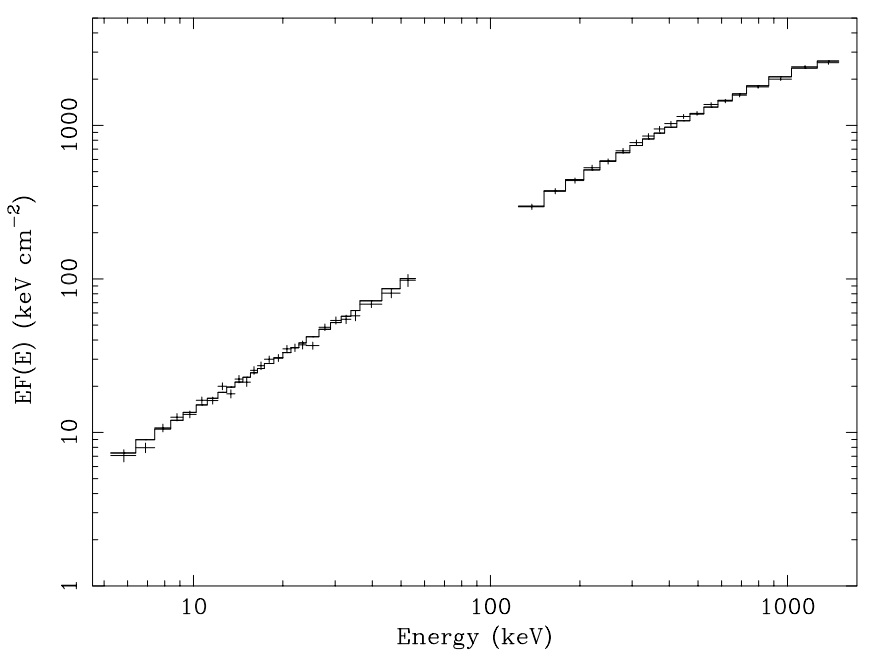

GRB 990705

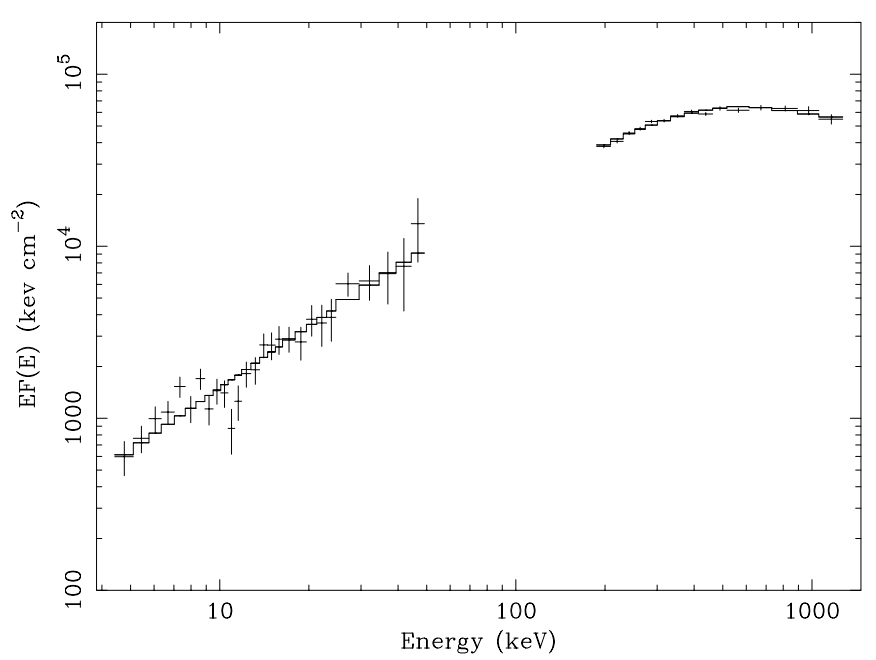

GRB 000214

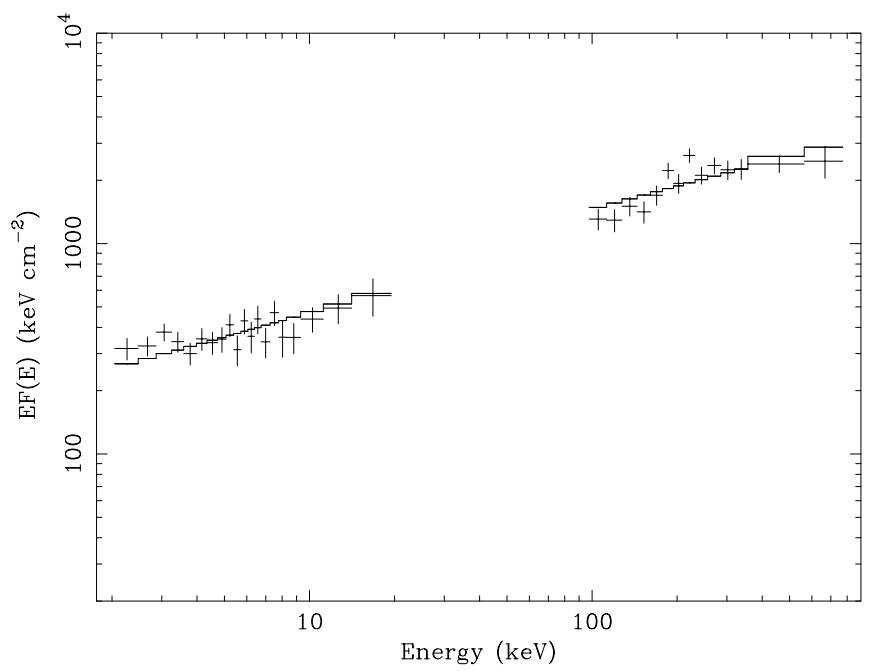

GRB 990510

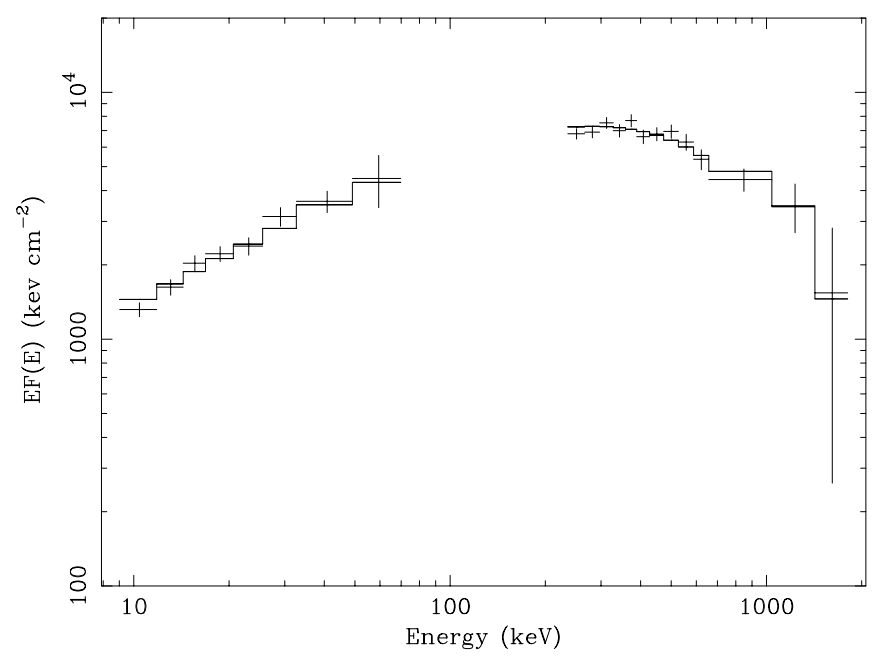

GRB 990712

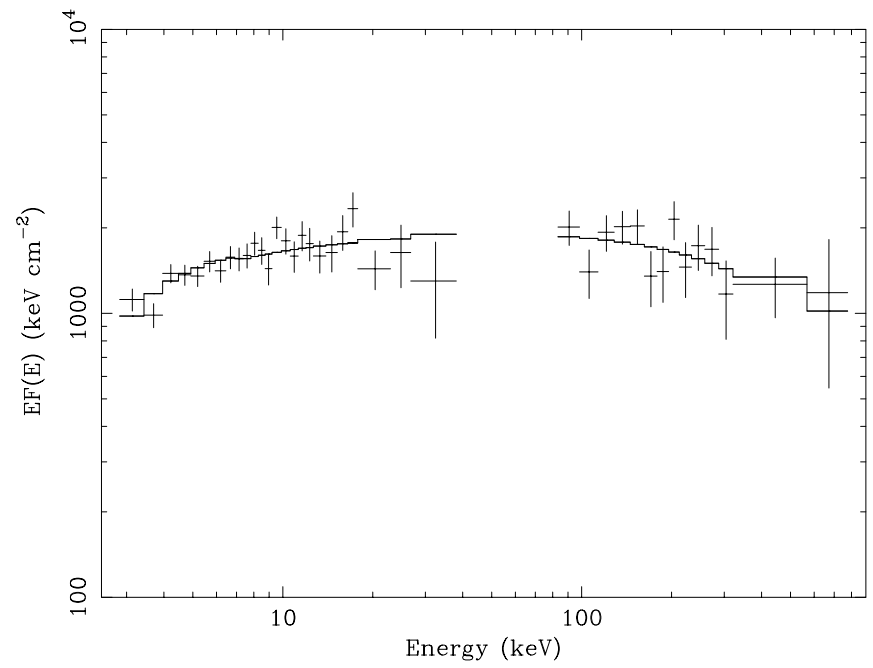

GRB 010222

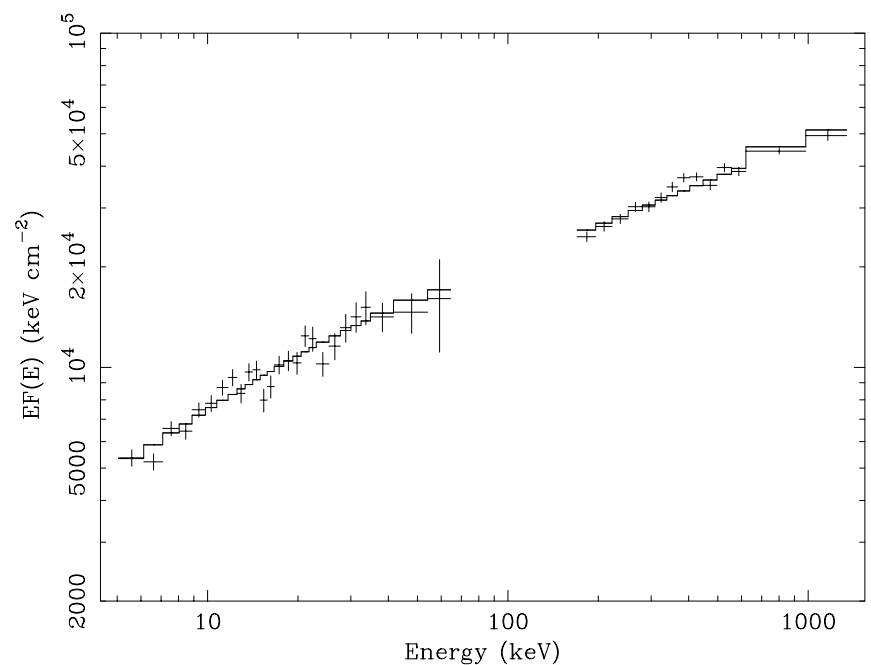

Fig. 1. continued. 
estimate of the intrinsic GRB properties. Instead of fitting the observed spectra, estimating the GRB fluences and then applying a $\mathrm{K}$-correction (method suggested by Bloom et al. 2001), we first blue-shifted the GRB spectra to the their cosmological rest frames ${ }^{1}$ and thus we derive their intrinsic shape. The total radiated energy of a GRB in a fixed energy range is then simply computed by integrating the bestfit model in that range (we adopt $1-10^{4} \mathrm{keV}$ ) and scaling for source luminosity distance. This latter quantity is derived assuming a flat Friedman-Robertson-Walker cosmological model with $H_{0}=65 \mathrm{~km} \mathrm{~s}^{-1} \mathrm{Mpc}^{-1}, \Omega_{\mathrm{m}}=0.3$, $\Omega_{\Lambda}=0.7$ (e.g. Carroll et al. 1992). For the sake of clarity, if $N\left(E, \alpha, E_{0}, \beta, A\right)$ is the best fit Band model to the timeintegrated and redshift-corrected spectrum of a GRB, $D_{\mathrm{L}}$ is its luminosity distance and $z$ its redshift, the $\left(1-10^{4} \mathrm{keV}\right)$ total radiated energy $E_{\text {rad }}$ is given by:

$E_{\mathrm{rad}}=\frac{\int_{1}^{10000} E N\left(E, \alpha, E_{0}, \beta, A\right) \mathrm{d} E \times 4 \pi D_{\mathrm{L}}^{2}}{(1+z)^{2}}$.

The $(1+z)^{2}$ factor comes out from the fact that the luminosity distance is defined in a way to account for the cosmological time dilation and spectral redshift when converting the observed source flux to the source luminosity (e.g. Coles \& Lucchin 1995), and both effects depend on $(1+z)$. Because we are dealing with fluences (i.e. the average flux multiplied to the GRB duration) and we have already corrected for spectral redshift before performing the spectral fitting, we have to divide by $(1+z)$ twice.

Finally, the correlation coefficients and the associated errors reported and discussed in the next sections were computed by properly weighing for data uncertainties (e.g. Bevington 1969) and verified with numerical simulations.

\section{Results}

Figure 1 shows the intrinsic time-integrated $E F(E)$ spectra of the GRBs included in our sample. All but one (GRB 000214) WFC plus GRBM redshift-corrected spectra are well described by the Band model. For GRB 000214, the spectrum is well fit by a simple power-law up to the high energy threshold of the GRBM; fitting this spectrum with the Band model only a $3 \sigma$ lower limit on $E_{\mathrm{p}}$ can be derived by fixing $\beta$ at -2.1 . The results of the fits are reported in Table 2, where the quoted uncertainties are $1 \sigma$ errors. For GRB 010222, $\beta$ is higher than -2 , thus we estimated a lower-limit for the peak energy by assuming $\beta=-2.1$. Table 2 also shows the total intrinsic released energies in the $1-10^{4} \mathrm{keV}$ range. For those events (namely, GRB 000214 and GRB 010222) for which $E_{\mathrm{p}}$ could not be determined, we derived the minimum and maximum

${ }^{1}$ If the spectral analysis is performed using the XSPEC package (Arnaud 1996), as we did, the correction of the spectra for cosmological redshift is obtained by multiplying by $(1+z)$ the ENERG_LO and ENERG_HI columns of the MATRIX extension and the E_MIN and E_MAX columns of the EBOUNDS extension of the response matrix FITS files. This task can be performed e.g. by using the $f v$ program belonging to the FTOOLS data reduction and FITS file manipulation package (Blackburn 1995).

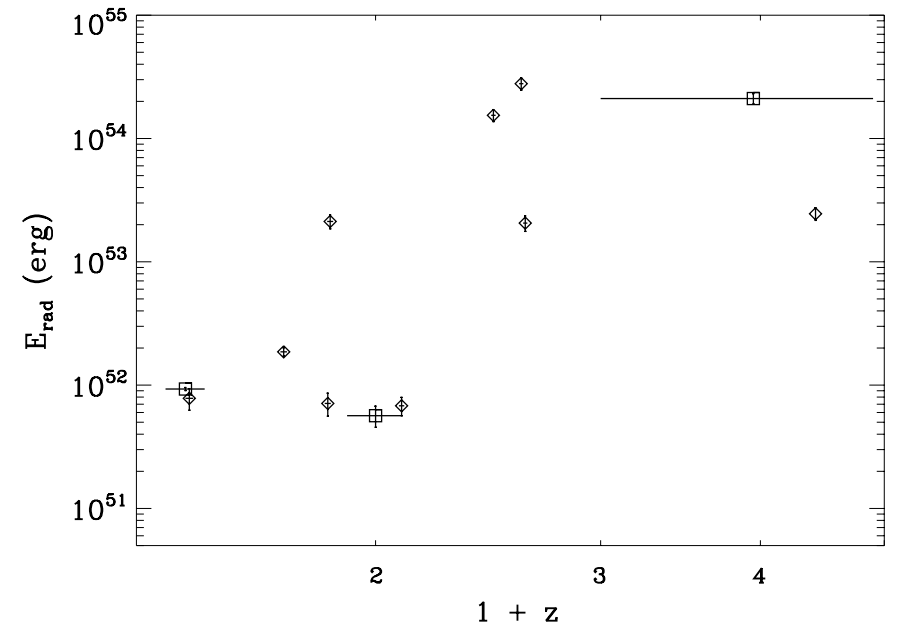

Fig. 2. Dependence on redshift of the isotropic total radiated energy. Diamonds indicate the 9 GRBs with firm redshift estimates, while squares refer to GRB 980326, GRB 980329 and GRB 000214.

values of $E_{\text {rad }}$ by assuming $E_{\mathrm{p}}$ equal to its lower limit and to $10000 \mathrm{keV}$, respectively (while keeping fixed $\alpha, \beta$ and the normalization A).

In Fig. 2 we show the total radiated energy $E_{\text {rad }}$ as a function of the redshift. Two important features can be inferred from these data: a) a general trend of $E_{\text {rad }}$ to increase with $z$ (see also Table 3 for the correlation coefficient), and b) the $E_{\text {rad }}$ values of most of the events in our sample are well above the sensitivity limit. We also find that the intrinsic $\mathrm{X}$ - and $\gamma$-ray durations, computed by correcting the observed durations (see Table 1) for the $(1+z)$ factor due to the cosmological time dilation, do not show significant correlations with redshift.

We performed a correlation study of the spectral parameters as a function of redshift and total radiated energy for the 9 GRBs in our sample with reliable redshift estimates. As clearly shown in Fig. 3, no correlation with redshift or $E_{\text {rad }}$ is found for the high energy photon index $\beta$ and the break energy $E_{0}$. However, we see an evidence of correlation with the redshift of the low energy photon index $\alpha$ and of the peak energy $E_{\mathrm{p}}$, and a clear correlation between $E_{\mathrm{p}}$ and $E_{\text {rad }}$. The correlation between $\alpha$ and $E_{\text {rad }}$ appears to be weaker. In Table 3 we report the correlation coefficients $r_{\log }$ between the logarithms of the quantities of interest. The correlation coefficients values between the direct quantities (not reported) are generally lower, indicating that the correlations found are better described by a power-law than a linear law. The most significant correlations are (see Table 3 ) between $\log |\alpha|$ and $\log (1+z)$ and between $\log E_{\mathrm{p}}$ and $\log E_{\mathrm{rad}}$. Both correlations remain strong $\left(r_{\log }=-0.842 \pm 0.108\right.$ and $-0.902 \pm 0.078$, respectively) even when the most distant event (GRB 971214), for the former, and the more energetic event (GRB 990123), for the latter, is excluded from the data set. Including the three events with less firm redshift determinations (GRB 980326, GRB 980329 and GRB 000214), assuming for each of them the redshift given by the centroid of the redshift interval reported in Table 1 , the $\log |\alpha|$ vs. $\log (1+z)$ correlation is confirmed $\left(r_{\log }=-0.859 \pm 0.078\right.$ for 12 events $)$ and the $\log E_{\mathrm{p}}$ vs. $\log E_{\mathrm{rad}}$ 
Table 2. Results of the Band model spectral fits of the $2-700 \mathrm{keV}$ spectra transformed in the cosmological GRB rest frames $\left({ }^{\star}\right)$ and derived total radiated energies assuming isotropic emission.

\begin{tabular}{|c|c|c|c|c|c|c|c|c|}
\hline GRB & $\begin{array}{l}\text { rest frame } \\
\text { band }(\mathrm{keV})\end{array}$ & $\alpha$ & $\beta$ & $\begin{array}{c}E_{0} \\
(\mathrm{keV})\end{array}$ & $\begin{array}{c}E_{\mathrm{p}}{ }^{a} \\
(\mathrm{keV})\end{array}$ & $A^{b}$ & $\chi^{2} /$ d.o.f. & $E_{\mathrm{rad}}{ }^{c}$ \\
\hline 970228 & $3.4-1186$ & $-1.54 \pm 0.08$ & $-2.5 \pm 0.4$ & $424 \pm 33$ & $195 \pm 64$ & $0.331 \pm 0.013$ & $\begin{array}{l}19.1 / 18 \\
\end{array}$ & $1.86 \pm 0.14$ \\
\hline 970508 & $3.7-1284$ & $-1.71 \pm 0.10$ & $-2.2 \pm 0.25$ & $502 \pm 150$ & $145 \pm 43$ & $0.074 \pm 0.015$ & $7.7 / 8$ & $0.71 \pm 0.15$ \\
\hline 971214 & $8.8-3094$ & $-0.76 \pm 0.17$ & $-2.7 \pm 1.1$ & $552 \pm 73$ & $685 \pm 133$ & $0.268 \pm 0.030$ & $20.7 / 17$ & $24.5 \pm 2.8$ \\
\hline 980326 & $4-1400$ & $-1.23 \pm 0.21$ & $-2.48 \pm 0.31$ & $92 \pm 20$ & $71 \pm 36$ & $0.160 \pm 0.032$ & $1.2 / 5$ & $0.56 \pm 0.11$ \\
\hline 980329 & $7.9-2765$ & $-0.64 \pm 0.14$ & $-2.2 \pm 0.8$ & $687 \pm 80$ & $935 \pm 150$ & $1.36 \pm 0.32$ & $26.5 / 24$ & $210.7 \pm 20.3$ \\
\hline 980613 & $4.2-1467$ & $-1.43 \pm 0.24$ & $-2.7 \pm 0.6$ & $342 \pm 170$ & $194 \pm 89$ & $0.072 \pm 0.012$ & $2.9 / 11$ & $0.68 \pm 0.11$ \\
\hline 990123 & $5.2-1820$ & $-0.89 \pm 0.08$ & $-2.45 \pm 0.97$ & $1828 \pm 84$ & $2030 \pm 161$ & $2.23 \pm 0.22$ & $21.5 / 41$ & $278.3 \pm 31.5$ \\
\hline 990510 & $5.2-1834$ & $-1.23 \pm 0.05$ & $-2.7 \pm 0.4$ & $549 \pm 26$ & $423 \pm 42$ & $0.897 \pm 0.091$ & $19.7 / 17$ & $20.6 \pm 2.9$ \\
\hline 990705 & $1.71-1290$ & $-1.05 \pm 0.21$ & $-2.2 \pm 0.1$ & $366 \pm 13$ & $348 \pm 28$ & $1.61 \pm 0.18$ & $46.5 / 36$ & $21.2 \pm 2.7$ \\
\hline 990712 & $2.9-1001$ & $-1.88 \pm 0.07$ & $-2.48 \pm 0.56$ & $779 \pm 125$ & $93 \pm 15$ & $0.223 \pm 0.013$ & $36.1 / 34$ & $0.78 \pm 0.15$ \\
\hline 000214 & $2.8-994$ & $-1.62 \pm 0.13$ & {$[-2.1]$} & $>308$ & $>117$ & $0.185 \pm 0.006$ & $57.1 / 37$ & $0.93 \pm 0.03$ \\
\hline 010222 & $4.9-1731$ & $-1.35 \pm 0.19$ & $-1.64 \pm 0.02$ & $146 \pm 41$ & $>886^{d}$ & $4.02 \pm 0.25$ & $51.6 / 41$ & $154.2 \pm 17.0$ \\
\hline
\end{tabular}

* For GRB 980326, GRB 980329 and GRB 000214 we assumed the central value of the redshift intervals reported in Table 1.

${ }^{a} \quad E_{\mathrm{p}}=E_{0} \times(2+\alpha)$.

${ }^{b}$ In photons $\mathrm{cm}^{-2} \mathrm{keV}^{-1}$.

$c$ Expressed in units of $10^{52} \mathrm{erg}$.

${ }^{d}$ Lower limit computed by assuming $\beta=-2.1$ (see also text).

correlation is even more significant $\left(r_{\log }=-0.941 \pm 0.037\right.$ for 10 events). The chance probability associated to these two correlations are $0.1 \%$ and $0.009 \%$ respectively. Assuming a power-law for the relationship between $|\alpha|$ and $(1+z)$ and between $E_{\mathrm{p}}$ and $E_{\text {rad }}$ we found the following best-fit slopes ( $1 \sigma$ uncertainties): $-0.78 \pm 0.18\left(\chi_{v}^{2}=0.54\right)$ and $0.52 \pm 0.06$ $\left(\chi_{v}^{2}=0.91\right)$, respectively. The bets fit results are shown in Fig. 4, where we have also included the values corresponding to GRB 980326, GRB 980329 and GRB 000214 and the lower limits on $E_{\mathrm{p}}$ from GRB 000214 and GRB 010222.

\section{Discussion}

We derived the intrinsic broad-band (from few $\mathrm{keV}$ to few $\mathrm{MeV}$, depending on source redshift) spectral properties and the total radiated energy in a fixed energy range $(1-10000 \mathrm{keV})$ of twelve BeppoSAX GRBs with known redshift. Thanks to the extension to $\mathrm{X}$-rays of the spectral analysis the determination of the low-energy spectral index of the Band function is less affected by the spectral curvature when the peak energy is around 100-200 keV. Determinations of the low-energy photon index from the BATSE data (e.g. Jimenez et al. 2001) could be affected by these curvature effects.

First of all we find a trend of the isotropically released energy $E_{\text {rad }}$ to increase with $(1+z)$ (see Fig. 2). This trend is intrinsic and not due to sensitivity limitations. In addition we find a statistically significant correlation between the peak energy $E_{\mathrm{p}}$ of the $E F(E)$ spectrum and the energy $E_{\mathrm{rad}}$. Also a correlation between the $\mathrm{X}$-ray photon index $\alpha$ and redshift is apparent. However we do not find any evidence of a correlation between peak flux, fluence or duration and redshift (see Table 1).
Three possible explanations of these features can be considered:

(1) given the still small number of GRBs with known redshift, our sample is biased and thus is not representative of the overall GRB population;

(2) selection effects due to the GRBM trigger logic and the GRBM + WFC combined sensitivities and data truncation effects may introduce biases in our results;

(3) the observed correlations are a manifestation of the intrinsic properties of the population of the long GRBs, which result to be brighter and more energetic (higher $E_{\mathrm{p}}$ ) at larger distances.

In principle, we cannot reject any of these hypotheses. However, in the next section we demonstrate that the selection effects, in case they are present, do not significantly influence our results.

\subsection{Selection and data truncation effects}

The evaluation of the biases due to selection effects and data truncation (e.g., the bias in the spectral parameters estimates due to the detector finite bandwidth) is a topical issue in the study of the correlations between different properties of GRBs, as discussed by various authors (e.g. Lloyd et al. 2000). For instance, the combined sensitivities and energy bands of the WFC and GRBM could favor the detection of harder and brighter GRBs at higher redshifts, thus originating the $E_{\text {rad }}$ vs. $(1+z)$ and the $E_{\mathrm{p}}$ vs. $E_{\text {rad }}$ relations that we found in our sample.

Feroci et al. (1999) performed an investigation on selection effects in a sample of $\sim 15$ GRBs detected with both GRBM and WFC with negative results, apart the selection of long GRBs ( $\geq 6 \mathrm{~s}$ ), as discussed above. The fluences of most GRBs in our sample are well above their minimum values as can be seen 


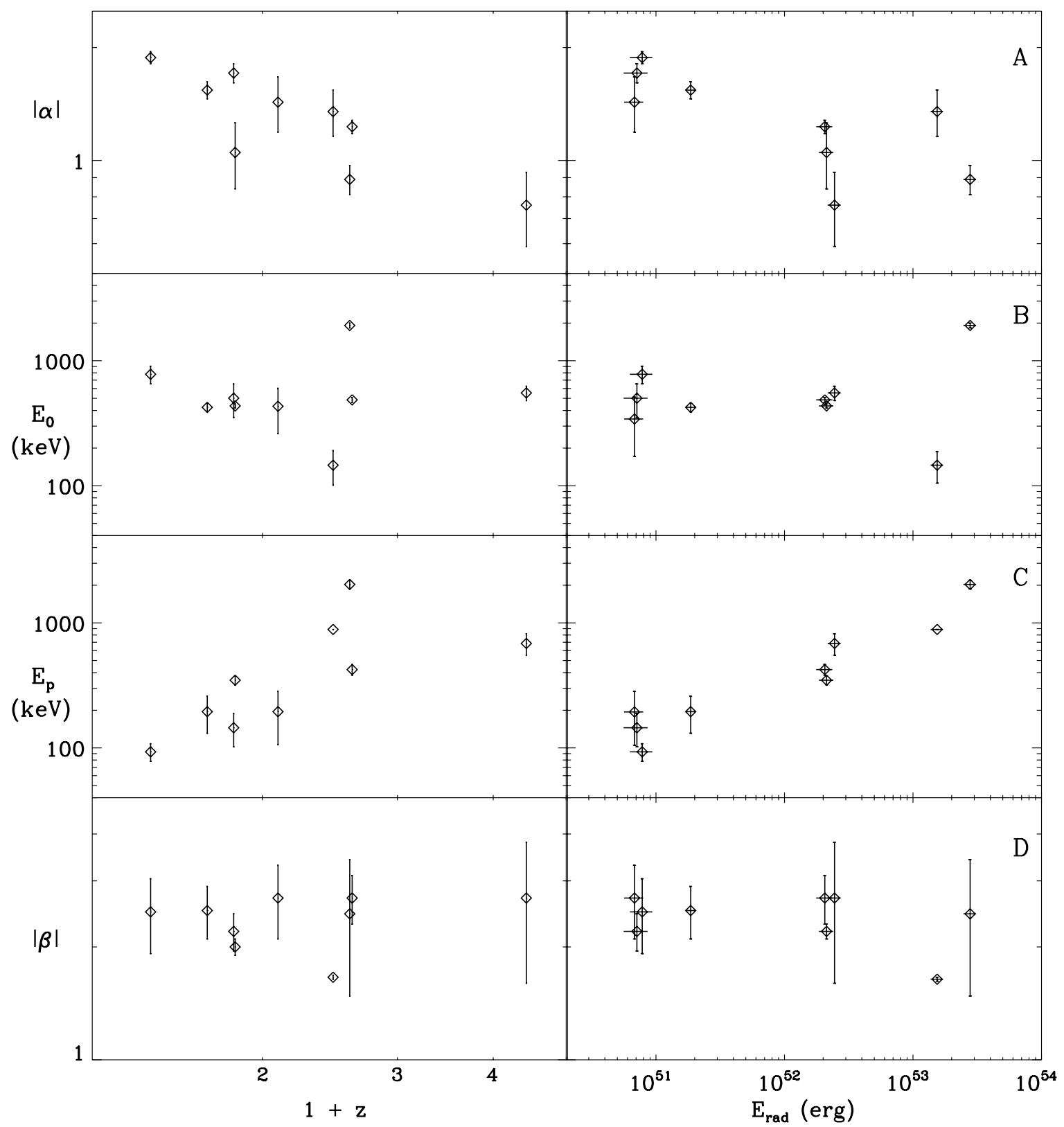

Fig. 3. Dependence on redshift (left panels) and isotropic total radiated energy (right panels) of the X-ray photon index a), break energy b), peak energy c) and gamma-ray photon index d) for the nine GRBs with firm redshift determinations.

Table 3. Correlation analysis results for the 9 GRBs with firm redshift estimates. The reported values and uncertainties have been computed following the method described in Sect. 3. The chance probabilities associated to the central values of the coefficients are given in parenthesis for the more significant correlations.

\begin{tabular}{lccc}
\hline \hline & $\log (1+z)$ & $\log E_{\mathrm{rad}}$ & $\log |\alpha|$ \\
\hline & & & \\
$\log E_{\mathrm{rad}}$ & $0.628 \pm 0.247(7.0 \%)$ & & \\
& & & \\
$\log |\alpha|$ & $-0.859 \pm 0.091(0.16 \%)$ & $-0.756 \pm 0.151(1.6 \%)$ & \\
& & & \\
$\log E_{\mathrm{p}}$ & $0.636 \pm 0.184(9.2 \%)$ & $0.949 \pm 0.036(0.005 \%)$ & $-0.821 \pm 0.075(0.44 \%)$ \\
\hline
\end{tabular}



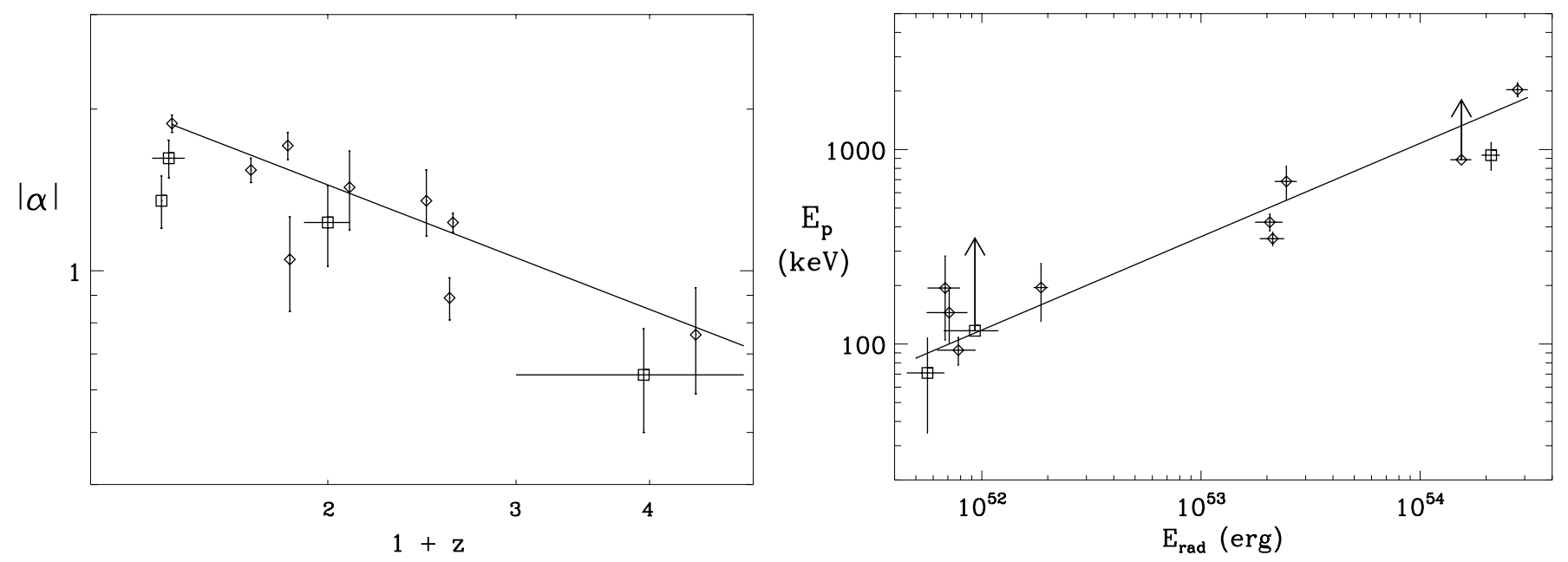

Fig. 4. Dependence on redshift of the X-ray photon index (left) and dependence on isotropic total radiated energy of $E_{\mathrm{p}}$ (right) for all the events included in the sample. The symbols are the same used for Fig. 1. The right panel also includes the lower limits on $E_{\mathrm{p}}$ for GRB 000214 and GRB 010222. In each panel we plotted over the data the power-law which best fits the values of the GRBs with firm redshift estimate (diamonds).

from Table 1 and Fig. 5, where the ratio between the measured GRB fluence in 40-700 $\mathrm{keV}, S_{\gamma}$, and its minimum detectable value $S_{\gamma, \min }$ is shown as a function of $(1+z)$. Also, no correlation is visible between the $S_{\gamma} / S_{\gamma \text {,min }}$ ratio and $(1+z)$. Even more distant GRBs have fluences much higher than the minimum ones, also taking into account the GRB shape as discussed in Sect. 2. Thus, we conclude that the observed trend between $E_{\mathrm{rad}}$ and $(1+z)$ is likely not affected by selection effects.

Somewhat more complex is the evaluation of possible data truncation biases in the correlations between spectral parameters and intensity or redshift. Lloyd \& Petrosian (1999) discuss extensively this topic and account for data truncation in their correlation analysis between $E_{\mathrm{p}}$ and bolometric fluence of a sample of bright BATSE events. Following a method similar to the one used by them, we computed for each GRB in our sample the minimum and maximum values of $E_{\mathrm{p}}$ and $\alpha$, the two spectral parameters for which we found significant correlation with $E_{\mathrm{rad}}$ and $(1+z)$, respectively. This is done by varying, first downward and then upward, the spectral parameter of interest while keeping fixed the other spectral parameters, the total radiated energy and the redshift, until the observer's rest-frame fluence reaches the minimum detectable value in the WFC 2$28 \mathrm{keV}$ or in the GRBM 40-700 keV energy band. Data truncation will affect significantly the correlation results if many values of the spectral parameters of interest are very close to their minimum or maximum value outside the acceptance range of other events. For the GRBs in our sample, none of the above conditions are verified, and thus our results appear not significantly biased by data truncation.

Finally, we note that the events in our sample are those for which an X-ray/optical follow-up has been performed. The follow-up observations were not performed by selecting special GRBs and thus we expect that no bias is introduced in the GRB sample selection.

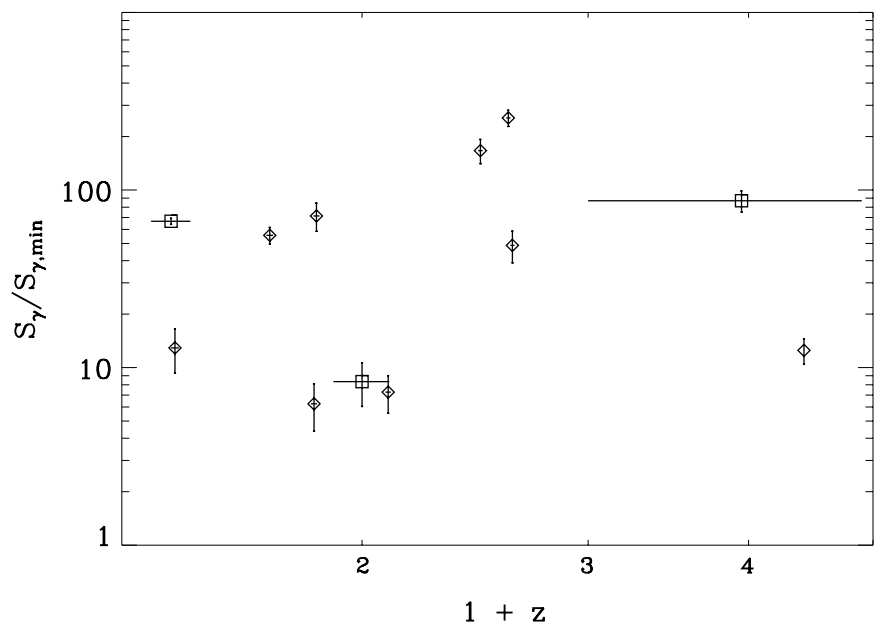

Fig. 5. Ratio between the measured gamma-ray $(40-700 \mathrm{keV})$ fluence and its minimum detectable value for each GRB in our sample, plotted as a function of redshift. Diamonds indicate the 9 GRBs with firm redshift estimates, while squares refer to GRB 980326, GRB 980329 and GRB 000214.

\subsection{Consequences of the found correlations}

The possibility of a positive correlation between GRBs peak energy $E_{\mathrm{p}}$ and bolometric fluence was already inferred by several studies, mainly based on BATSE detected events (e.g. Mallozzi et al. 1995; Brainerd 1997), which suffered the lack of the knowledge of their redshift (Lloyd et al. 2000). Our finding of an $E_{\mathrm{p}}$ vs. $E_{\text {rad }}$ correlation confirms the previous results, with the advantage that the redshifts of the GRBs in our sample are known. We find that $E_{\mathrm{p}}$ depends on $E_{\text {rad }}$ accordingly to the following relationship:

$E_{\mathrm{p}} \propto E_{\mathrm{rad}}^{0.52 \pm 0.06}$.

As discussed by Lloyd et al. (2000), a similar power law relation $\left(E_{\mathrm{p}} \propto \epsilon_{\mathrm{rad}}^{0.5}\right)$ is expected in the case of an Optically Thin 
Synchrotron Shock Model (OTSSM) for an electron distribution with a power-law shape $\left(N(\gamma)=N_{0} \gamma^{-\mathrm{p}}\right.$ for $\left.\gamma>\gamma_{\mathrm{m}}\right)$ with $\gamma_{\mathrm{m}}$, GRB duration and $N_{0}$ invariant from burst to burst. We know that these assumptions are not completely true (e.g. GRB duration changes from burst to burst) and $E_{\mathrm{rad}}$ could be lower if the emitted radiation is beamed, but this result should be taken into account in working-out GRB emission models.

The interpretation of the other found relationship:

$|\alpha| \propto(1+z)^{-0.78 \pm 0.18}$

is not straightforward.

The fact that most of the low-energy photon indices $\alpha$ are in the range from $-2 / 3$ (instantaneous index predicted by the OTSSM; e.g., Tavani 1996) and -3/2 (index predicted for synchrotron cooled leptons; e.g., Cohen et al. 1997) is a strong hint in favor of the the synchrotron as primary emission model. However Eq. (2) would suggest that radiative cooling occur more actively in GRBs with relatively small redshift. Apart the fact that this evolutionary effect is difficult to justify, we have investigated other possible origin of the relation (2). As a result, we have found that it does not reflect a physical property of GRBs but is a consequence of other correlations. In fact, also an evidence of correlation between $\alpha$ and $E_{\mathrm{p}}$ was found (see Table 3). This evidence was also found for BATSE GRBs and was explained (e.g., Lloyd and Petrosian 2000) as due partially to the dependence of the $\alpha$ estimate on the spectral curvature nearby $E_{\mathrm{p}}$ and partially to the fact that $E_{\mathrm{p}}=(2+\alpha) \times E_{0}$ (Band et al. 1993). Taking into account the other found correlations between $E_{\mathrm{p}}$ and $E_{\mathrm{rad}}$ and between $E_{\mathrm{rad}}$ and $(1+z)$, the expected relationship between $\alpha$ and $(1+z)$ is given by $|\alpha|=(2.76 \pm 0.09) \times(1+z)^{-0.75 \pm 0.06}$, with a power-law index which is fully consistent with the best fit power-law index of Eq. (2).

Acknowledgements. This research was partly supported by the Italian Space Agency (ASI). We thank the teams of the BeppoSAX Operative Control Center and Scientific Data Center for their efficient and enthusiastic support to the GRB alert program.

\section{References}

Amati, L., Frontera, F., Costa, E., et al. 1999a, A\&AS, 138, 403
Amati, L. 1999b, Ph.D. Thesis, available at http://tonno.tesre.bo.cnr.it/ amati/tesi/tesi.html Amati, L., Frontera, F., Vietri, M., et al. 2000, Science, 290, 953

Andersen, M. I., Hjorth, J., Gorosabel, J., et al. 2002, in preparation

Antonelli, L. A., Piro, L., Vietri, M., et al. 2000, ApJ, 545, L39

Arnaud, K. A. 1996, in Astronomical Data Analysis Software and Systems V, ed. J. Jacoby, \& J. Barnes, ASP Conf. Ser., 101, 17

Band, D., Matteson, J., Ford, L., et al. 1993, ApJ, 413, 281

Bevington, J. R. 1969, in Data reduction and error analysis for the physical sciences (McGraw-Hill)

Blackburn, J. K. 1995, in Astronomical Data Analysis Software and Systems IV, ed. R. A. Shaw, H. E. Payne, \& J. J. E. Hayes (San Francisco: ASP), ASP Conf. Ser., 77, 367

Bloom, J. S., Djorgovski, S. G., Kulkarni, S. R., \& Frail, D. A. 1998, ApJ, 507, L25

Bloom, J. S., Kulkarni, S. R., Djorgovski, S. G., et al. 1999, Nature, 401, 453

Bloom, J. S., Frail, D. A., \& Sari, S. R. 2001, AJ, 121, 2879

Boella, G., Butler, R. C., Perola, G. C., et al. 1997, A\&AS, 122, 299

Carroll, S. M., Press, W. H., \& Turner, E. L. 1992, ARA\&A, 30, 499

Cohen, E., Katz, J. L., Piran, T., et al. 1997, ApJ, 488, 330

Coles, P., \& Lucchin, F. 1995, in Cosmology. The origin and evolution of cosmic structure (John Wiley \& Sons)

Dickey, J. M., \& Lockman, F. J. 1990, ARA\&A, 28, 215

Feroci, M., Preger, B., Costa, E., et al. 1999, A\&AS, 138, 407

Frontera, F., Costa, E., Dal Fiume, D., et al. 1997, A\&AS, 122, 357

Frontera, F., Amati, L., Costa, E., et al. 2000, ApJS, 127, 59

Guidorzi, C. 2002, Ph.D. Thesis, University of Ferrara

Kulkarni, S. R., Djorgovski, S. G., Ramaprakash, A. N., et al. 1998, Nature, 393, 35

Kulkarni, S. R., Djorgovski, S. G., Odewahn, S. C., et al. 1999, Nature, 398, 389

Jager, R., Mels, W. A., Brinkman, A. C., et al. 1997, A\&AS, 125, 557

Jimenez, R., Band, D., \& Piran, T. 2001, ApJ, 561, 171

Lamb, D. Q., Castander, F. J., Reichart, D. E., et al. 1999, A\&AS, 138, 479

Lloyd, N. M., \& Petrosian, V. 1999, ApJ, 511, 550

Lloyd, N. M., Petrosian, V., \& Mallozzi, R. S. 2000, ApJ, 534, 227

Morrison, R., \& McCammon, D. 1983, ApJ, 270, 119

Stanek, K. Z., Garnavich, P., Jha, S., \& Pahre, M. 2001, IAU Circ., No. 7586

Tavani, M. 1996, Phys. Rev. Lett., 76, 3478

Tavani, M. 1997, ApJ, 497, L21

Vreeswijk, P. M., Fruchter, A. S., Kaper, L., et al. 2000, ApJ, 546, 672 